\title{
Transfer of the magnetic axis of an undulator to mechanical fiducial marks of a Laser Tracker System
}

\author{
Bora Ketenoğlu ${ }^{\mathrm{a}, \mathrm{b}}$, Uwe Englisch ${ }^{\mathrm{b}}$, Yuhui Li $^{\mathrm{b}}$, Frederik Wolff-Fabris ${ }^{\mathrm{b}}$, Wolf Benecke ${ }^{\mathrm{c}}$, \\ Martin Noak ${ }^{\mathrm{c}}$, Johannes Prenting ${ }^{\mathrm{c}}$, Markus Schloesser ${ }^{\mathrm{c}}$ and Joachim Pflueger ${ }^{\mathrm{b}, \mathrm{d}}$ \\ ${ }^{a}$ Department of Engineering Physics, Ankara University, Tandoğan, Ankara 06100, Turkey \\ ${ }^{b}$ European XFEL, Albert-Einstein-Ring 19, Hamburg 22761, Germany \\ ${ }^{c}$ Deutsches Elektronen-Synchrotron (DESY), Notkestrasse 85, Hamburg 22607, Germany \\ ${ }^{d}$ Corresponding Author: joachim.pflueger@xfel.eu; Tel +494089983242, Fax +494089943242
}

Keywords: Magnetic alignment, magnetic fiducialization, Undulator magnetic axis

\begin{abstract}
The exact geometric location of the magnetic centers of sensors or sensor systems using Hall probes or pick-up coils is usually not known with high precision. In order to transfer the high spatial accuracy of magnetic measurements to external mechanic fiducials a device called "Magnetic Landmark" was developed and is described in this report. Its purpose is to establish the exact relation between 'magnetic' coordinates used on magnetic measurement systems and 'mechanic' coordinates used for alignment.

The landmark consists of a permanent magnet configuration, which generates a field distribution with well-defined zero crossings in two orthogonal directions, which can be exactly localized with micrometer precision using magnetic measurement systems. For the 'mechanic' measurements several redundant monuments for laser fiducials can be used.

Using flip tests for the magnetic as well as mechanic measurements the center positions are determined in magnetic and mechanic coordinates. Using them the relation between the magnetic and surveying coordinates can be established with high accuracy.

This report concentrates on the description of the landmark. A thorough analysis on achievable accuracy is presented. The method was developed for the alignment of the 91 undulator segments needed for the European XFEL but can be applied to other magnet systems as well.
\end{abstract}

\section{Introduction}

The magnetic axes of individual undulator segments in long X-ray FELs such as the European XFEL (EXFEL) or Linac Coherent Light Source (LCLS ) need to be aligned with an accuracy of typically $\pm 50-100 \mu \mathrm{m}$ or better $/ 1,2 /$. This puts challenging requirements on magnetic measurements and geometric alignment.

State of the art magnetic measurements allow for high spatial resolution. As an example, the exact location of the magnetic center axis in an undulator can be measured with an accuracy of $\pm 5-10 \mu \mathrm{m}$ in both transverse directions. It is, however, difficult to transfer this magnetic accuracy to geometric fiducials, which can be used for in situ alignment in the tunnels.

The basic problem is that the exact geometric location of the magnetic center of a sensor or a sensor system is only known to an estimated accuracy of \pm 0.5 - $1 \mathrm{~mm}$ at best and moreover may slightly drift in time due to unwanted changes in the mechanical supports. In order to make full use of the spatial accuracy of the magnetic measurements a method is needed which transfers the magnetic coordinates to externally accessible fiducials for geometric alignment methods such as laser trackers. For LCLS a method was described /3,4/ which makes use of two "Fiducialization Magnets", which are temporarily attached to the ends of a $3.3 \mathrm{~m}$ long fixed gap undulator segment. A fiducialization magnet consists of two magnetic needles, which produce well-defined field distributions directly related to laser tracker monuments. For the transfer of the magnetic coordinates the undulator needs to be placed on a 3D coordinate measurement machine, which due to the compact size of the fixed gap LCLS undulator segments is straight forward to do.

For the EXFEL an alternative method is required since the undulator segments are gap adjustable and have a substantial size of 5 by 2.4 by 1.5 meters. A device internally called "Magnetic Landmark" was developed, 
which allowed the transfer from magnetic to geometric coordinates using state of the art Laser Trackers. It is described in this paper.

\section{Detailed description}

\subsection{Basic principle}

For the magnetic landmark described in this paper flip tests play a key role. The principle is shown in figure 1. The Y-center position of an object is determined by measuring the Y position of an object point, marked by the red cross, in two orientations, one, $Y_{N}$, at $0^{\circ}$ and the other, $Y_{F}$, at $180^{\circ}$. The center position, $Y_{C}$, of the object is determined by:

$$
Y_{C}=\left(Y_{N}+Y_{F}\right) / 2
$$

The Z-center positions are obtained accordingly. This principle can be applied to magnetic or mechanic objects and the results will be in mechanic or magnetic coordinates. For the flip test there must be provisions to place the object reproducibly in both orientations.

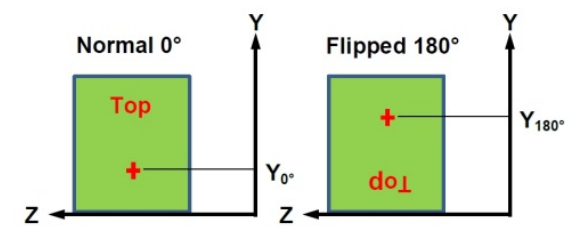

Figure 1: Principle of a flip test

An excellent mechanical object point is the monument for a laser retro-reflector, which can be placed such that it can be measured in $0^{\circ}$ and $180^{\circ}$ positions.

\subsection{Magnetic system}

For the definition of a magnetic object point a suitable configuration of permanent magnets (PMs) is needed. Its field distribution should have zero crossings, which can be localized precisely. First ideas were presented in $/ 5 /$. A configuration with this property for the $\mathrm{Y}$ component of the magnetic field, $B_{Y}$, is shown in figure 2 a) together with the definition of the coordinate system and its origin used in this paper. Low cost cubic shaped permanent magnets (PMs) made of NdFeB with a remanent field of $1.2 \mathrm{~T}$ and edge-length of $20 \mathrm{~mm}$ are used. Bottom and top magnets are separated by a gap of $12 \mathrm{~mm}$. The left group is separated by the right pair by $10 \mathrm{~mm}$. The exact dimensions are given here for information. In the median plane this magnet configuration has well defined zero crossings. Their Y and Z-coordinates can be localized precisely in normal and $180^{\circ}$ flipped state as will be described in more detail below.

\section{a) Normal}

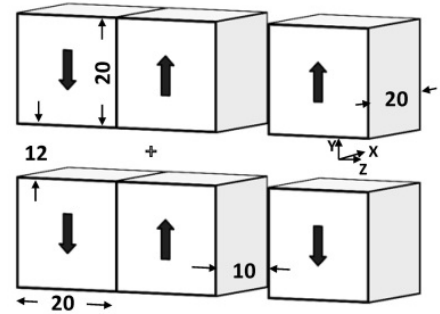

\section{b) $180^{\circ}$ Flipped}

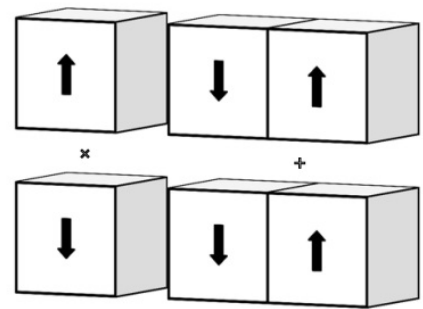

Figure 2: Configuration of cubic PM blocks.

a): Normal position. In the median plane the left four blocks form a normal quadrupole with the $\mathrm{Z}$ center position at the position of the cross. In Y direction the rightmost two blocks form a skew quadrupole. Its center defines the coordinate origin. b):For the flip test the whole magnet configuration is rotated by $180^{\circ}$ around the $\mathrm{X}$-Axis in the coordinate origin.

This PM configuration is a combination of a normal and a skew planar PM quadrupole, which were first proposed by Tatchyn /6/. The leftmost four magnets in figure 2a) form the normal PM Quadrupole. Near its center marked by the cross the $\mathrm{B}_{\mathrm{y}}$ field component has a linear dependence in $\mathrm{z}$.

$$
B_{y}(z)=c_{N o r m} \cdot\left(z-z_{N o r m}\right)
$$


Here $c_{\text {Norm }}$ is the normal gradient. At the center position, $z_{N o r m}$, the sign of the $B_{Y}$ component changes. Near $\mathrm{x}, \mathrm{y}=0$ this point can be localized with high precision in magnetic measurements by scanning $\mathrm{B}_{\mathrm{y}} \mathrm{vs}$. $\mathrm{z}$.

The magnet pair on the right with anti-parallel magnetization is a planar PM skew quadrupole. The field near its center, which is the coordinate origin as well is described by:

$$
B_{y}(y)=c_{\text {Skew }} \cdot\left(y-y_{\text {Skew }}\right)
$$

Here $\mathrm{c}_{\text {Skew }}$ is the skew gradient and $\mathrm{y}_{\text {skew }}$ the exact $\mathrm{y}$-position, where the $\mathrm{B}_{\mathrm{y}}$ component changes sign. In complete analogy to $z_{\text {Norm }} y_{\text {Skew }}$ is localized by scanning $\mathrm{B}_{\mathrm{y}}$ vs. $\mathrm{y}$ near $\mathrm{y}, \mathrm{z}=0$. The two zero crossings $\mathrm{z}_{\text {Norm }}$ and $\mathrm{y}_{\text {Skew }}$ define the magnetic object point in the $\mathrm{Y}-\mathrm{Z}$ plane near $\mathrm{x}=0$.

The magnetic $\mathrm{Y}$ and $\mathrm{Z}$ center positions are obtained by a flip test, i.e. by flipping the magnet array around the $\mathrm{X}$ axis in the origin as sketched in figure $2 b$ ). Now there are positions in normal and flipped state, which can be readily measured with the magnetic sensor sensitive to $B_{y}$ : The magnetic centers are obtained using Eq. (1).

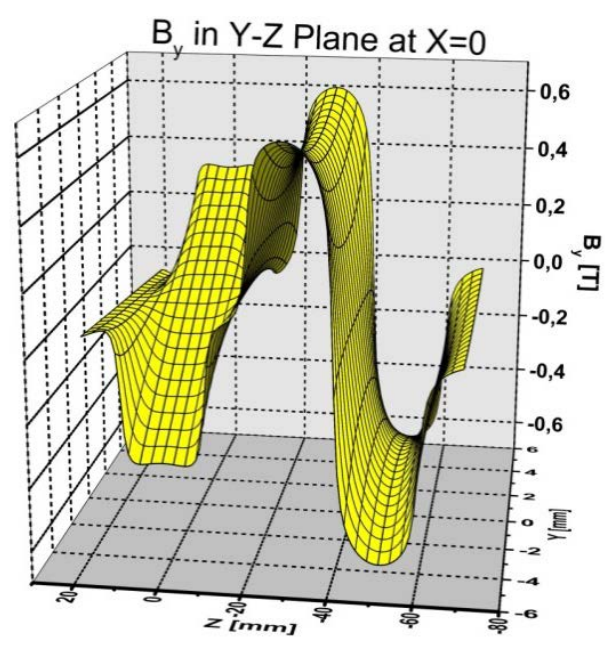

Figure 3: $\mathrm{B}_{\mathrm{y}}$ component in the $\mathrm{Y}-\mathrm{Z}$ plane at $\mathrm{X}=0$ for the normal $0^{\circ}$ state shown in figure $2 \mathrm{a}$ ).

Note the different scales in $\mathrm{Y}$ and $\mathrm{Z}$

Figure 3 shows the distribution of the $\mathrm{B}_{\mathrm{y}}$ component in the $\mathrm{Y}-\mathrm{Z}$ plane at $\mathrm{X}=0$ for the magnet configuration shown in Figure 2a). Note, for better visibility the z-axis direction has been reversed from right to left. The skew quadrupole is seen on the left around $\mathrm{z}=0 \mathrm{~mm}$ by the linear increase along Y. Similarly the normal quadrupole is seen around $\mathrm{z}=-40 \mathrm{~mm}$ by the steep linear increase along $\mathrm{z}$. Note the different scales of $\mathrm{y}$ and $\mathrm{z}$.

Figure 4 demonstrates the sensitivity to misalignment in the orthogonal directions. In figure 4a) the exact value of the $\mathrm{Y}$ coordinate of the zero crossing under the skew quadrupole at $\mathrm{z}=0 \mathrm{~mm}$ is shown. It is seen that in the
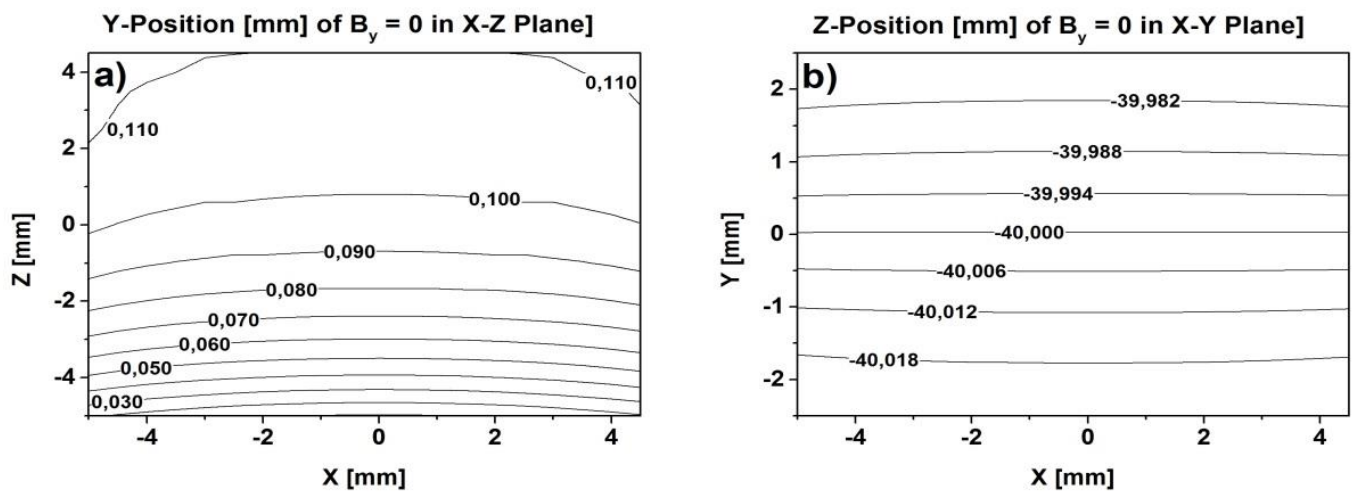

Figure 4: Sensitivity of the zero crossing of the $B_{y}$ component to misalignment.

a) the $Y$ coordinate for $B_{y}=0$ under the skew quadrupole in the $X-Z$ plane near $Y=0 \mathrm{~mm}$

b) the $\mathrm{Z}$ coordinate for $\mathrm{B}_{\mathrm{y}}=0$ under the normal quadrupole in the $\mathrm{X}-\mathrm{Y}$ plane near $\mathrm{Z}=-40 \mathrm{~mm}$

For very moderate alignment the zero crossings are preserved. For more details see text. 
whole plotted area in the $\mathrm{X}-\mathrm{Z}$ plane the $\mathrm{Y}$ zero crossing varies only by $80 \mu \mathrm{m}$ in total and much less if the area is reduced. For \pm 0.5 by $\pm 0.5 \mathrm{~mm}^{2}$, which is a moderate alignment accuracy, the variation is estimated to $<10 \mu \mathrm{m}$ in total. Similarly figure $4 \mathrm{~b}$ ) shows the exact value of the Z-zero crossing in the plotted X-Y plane under the normal quadrupole located at $\mathrm{z}=-40 \mathrm{~mm}$. It varies in total only by $36 \mu \mathrm{m}$. For \pm .5 by $\pm 0.5 \mathrm{~mm}^{2}$ the total variation is $12 \mu \mathrm{m}$ only. This demonstrates that there is only moderate dependence of the zero crossings to misalignment in the orthogonal directions.

\section{Mechanic design and application}

A Magnetic Landmark following the design principles as described above was built and is shown in figure 5. For orientation the coordinate system is shown as well. The magnet design and dimensions described in section 2.2 are used. The transverse size of the landmark in $\mathrm{Z}$ direction is about $140 \mathrm{~mm}$. The C-shaped support is made of non-magnetic brass. Magnets are positioned by conical fastener clamps. The geometry allows scanning in the $Y$ direction over several millimeters and without limits along the $\mathrm{Z}$ direction. For the mechanical measurements there are 14 labeled conical recessions which are distributed symmetrically around the device. These are the monuments for the retro reflector of a laser tracker system. Their high redundancy facilitates accuracy but also visibility so that at least some are in the line of sight for the laser tracker.

Components used for surveying equipment increase precision and facilitate alignment: Two triple-self centering mounts, one on either side, are symmetrically attached, as seen in figure 5a). They fit into a precision adjustable Wild/Leica tribrach as shown in figure $5 b$ ) in the unflipped state. The $180^{\circ}$ flipped state is shown in figure $5 \mathrm{c}$ ). For demonstration the directions of the magnetic moments of the magnets in agreement with figure 2a) and b) are shown by the arrows in figure $5 b$ ) and c).

The whole Magnetic Landmark assembly with the tribrach can be attached and aligned on a support stand in a similar fashion as an optical alignment tool and placed at a convenient location.

Using the magnetic landmark is straight forward. The first step is to determine the mechanical center in $\mathrm{Y}$ and $\mathrm{Z}$ using the laser tracker monuments in analogy to Eq. (1):

$$
Y, Z_{\text {Cent }, \text { Mech }}=\frac{Y, Z_{M e c h, N}+Y, Z_{M e c h, F}}{2}
$$

Here a single or an average over several of the redundant monuments may be used for the laser measurements. Like in Eq.(1) $\mathrm{N}$ stands for "normal”, $0^{\circ}$ and $\mathrm{F}$ for "Flipped" $180^{\circ}$.In the second step the magnetic center is
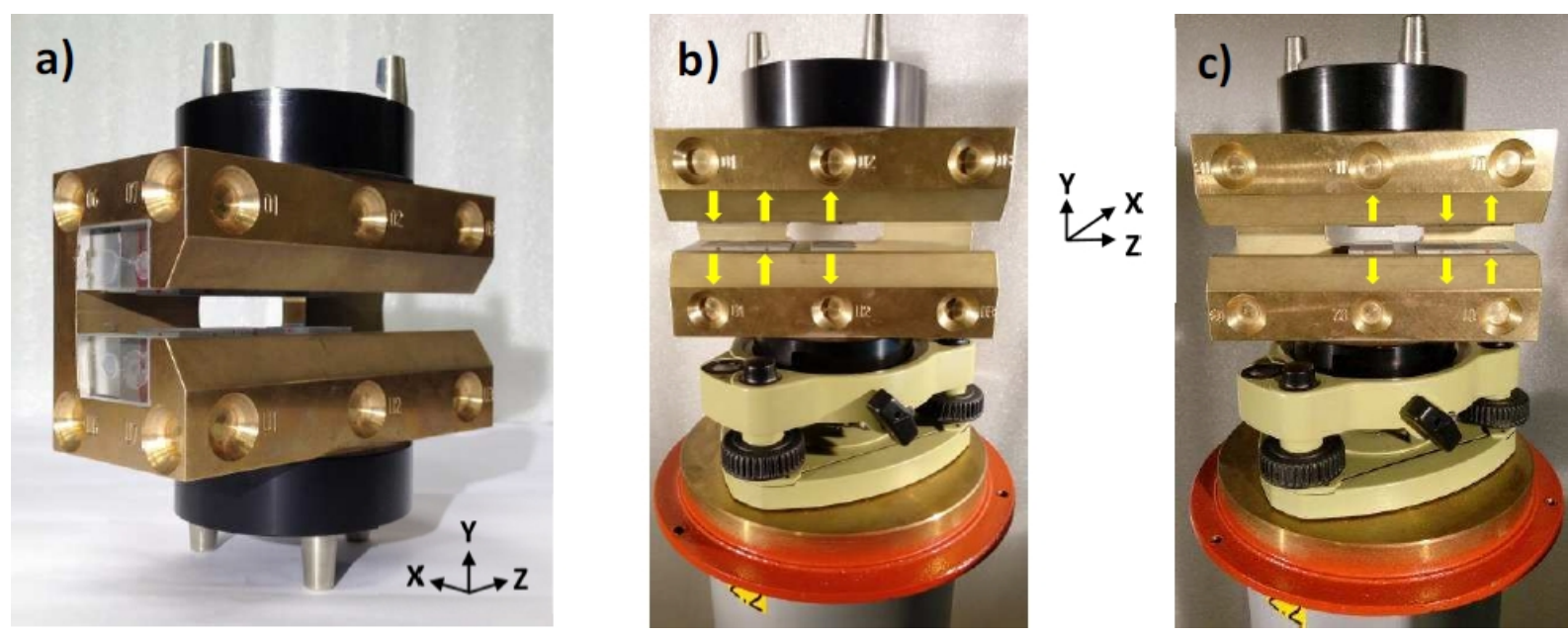

Figure 5: Implementation of the Magnetic Landmark. A massive non-magnetic C-frame supports the magnets and provides stability. a) Highly symmetric triple conical supports with self-centering studs symmetrically attached on top and bottom facilitate flip tests. b) A Wild/Leica Tribrach is used together with the self-centering studs. It allows high precision alignment and a very reproducible insertion for the $0^{\circ}$ and $180^{\circ}$ flip around the $\mathrm{X}$-axis. The position shown is the $0^{\circ}$ normal state.

d) Landmark inserted in the $180^{\circ}$ flipped state.

Only the tips of the PMs are visible. The magnet orientation is shown by the arrows for demonstration. A number of redundant labeled recessions are distributed over the landmark where a Laser Retro reflector monument can be placed for the mechanic measurements.

determined using magnetic measurements. To do so magnetic field scans of $\mathrm{B}_{\mathrm{y}}$ in y direction over about $\pm 0.3 \mathrm{~mm}$ around the zero crossing and over about -70 to $+60 \mathrm{~mm}$ in z-direction are made in $0^{\circ}$ and $180^{\circ}$ position as shown in figure $5 b$ ) and c). Figure 6 a) shows the two Y-scans of $B_{y}$ at the location of the skew quadrupole array in 
normal (blue, dashed) and flipped (red, full) orientation. $\mathrm{B}_{\mathrm{Y}}$ shows very good linear dependency. The zero crossings can be exactly determined to be at $-0.1523 \mathrm{~mm}$ and $0.0443 \mathrm{~mm}$ for the $0^{\circ}$ unflipped and $180^{\circ}$ flipped state, respectively. In a similar fashion the Z-scans shown in figure $6 \mathrm{~b}$ ) result in the zero crossings at -42.928 for the $0^{\circ}$ unflipped and $37.538 \mathrm{~mm}$ for the $180^{\circ}$ flipped orientation. Again in analogy to Eq. (1) the magnetic centers in the coordinate system of the magnetic bench are obtained:

$$
Y, Z_{\text {Cent }, \text { Mag }}=\frac{\left(Y, Z_{M a g, N}+Y, Z_{M a g, F}\right)}{2}
$$

Using the numbers given above the magnetic center is at: $\mathrm{Y}_{\mathrm{Cent}, \mathrm{Mag}}=-0.054 \mathrm{~mm}$ and $\mathrm{Z}_{\mathrm{Cent}, \mathrm{Mag}}=-2.695 \mathrm{~mm}$. Error estimates will be given below.The small values are not surprising since the landmark is placed on purpose near the expected magnetic axis.

Since the center of a flip test is the same for magnetic or mechanic measurements the offsets between the mechanic coordinates obtained by Laser Tracker and the magnetic bench coordinates are given by:

$$
\mathrm{Y}, \mathrm{Z}_{\text {Mech-Mag }}=\mathrm{Y}, \mathrm{Z}_{\text {Cent,Mech }}-\mathrm{Y}, \mathrm{Z}_{\text {Cent,Mag }}
$$
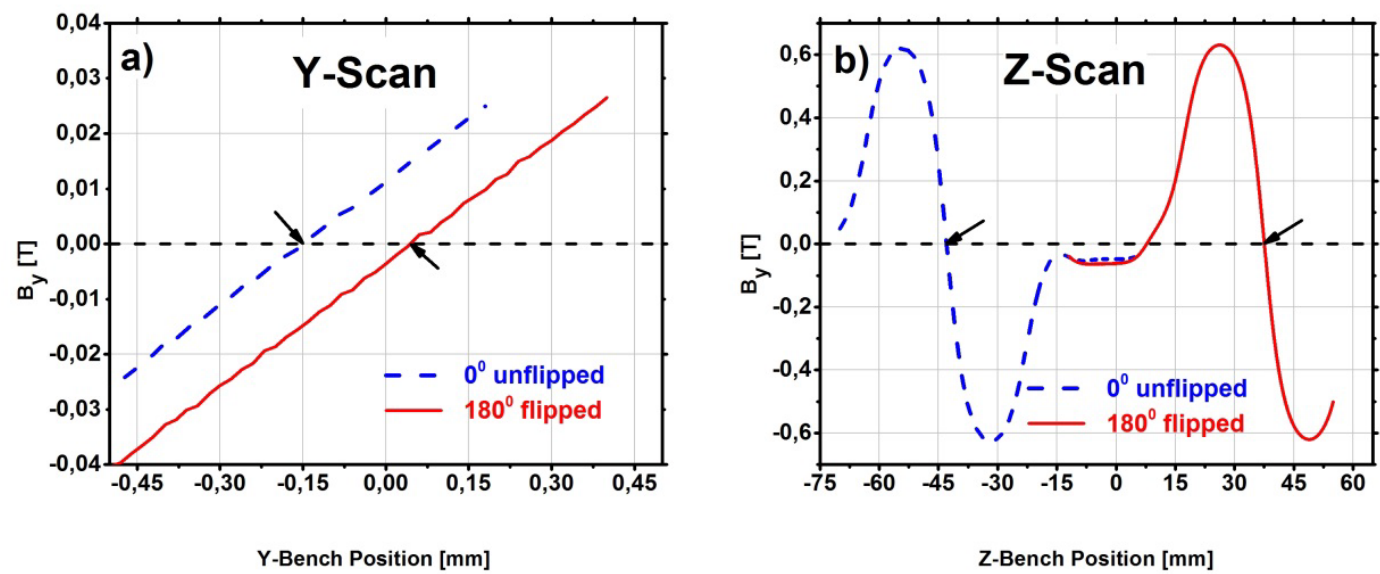

Figure 6: Results of magnetic scans in in normal (blue, dashed) and flipped (red, solid) orientation. The relevant zero crossings are marked with arrows: a) Scans of $B_{Y}$ vs $y$; b) Scans of By vs z.

\section{Application example}

A practical application example of the landmark is the fiducialization of undulators with respect to their magnetic axis. It is described below. Its use is, however, quite general and can be extended to the alignment of

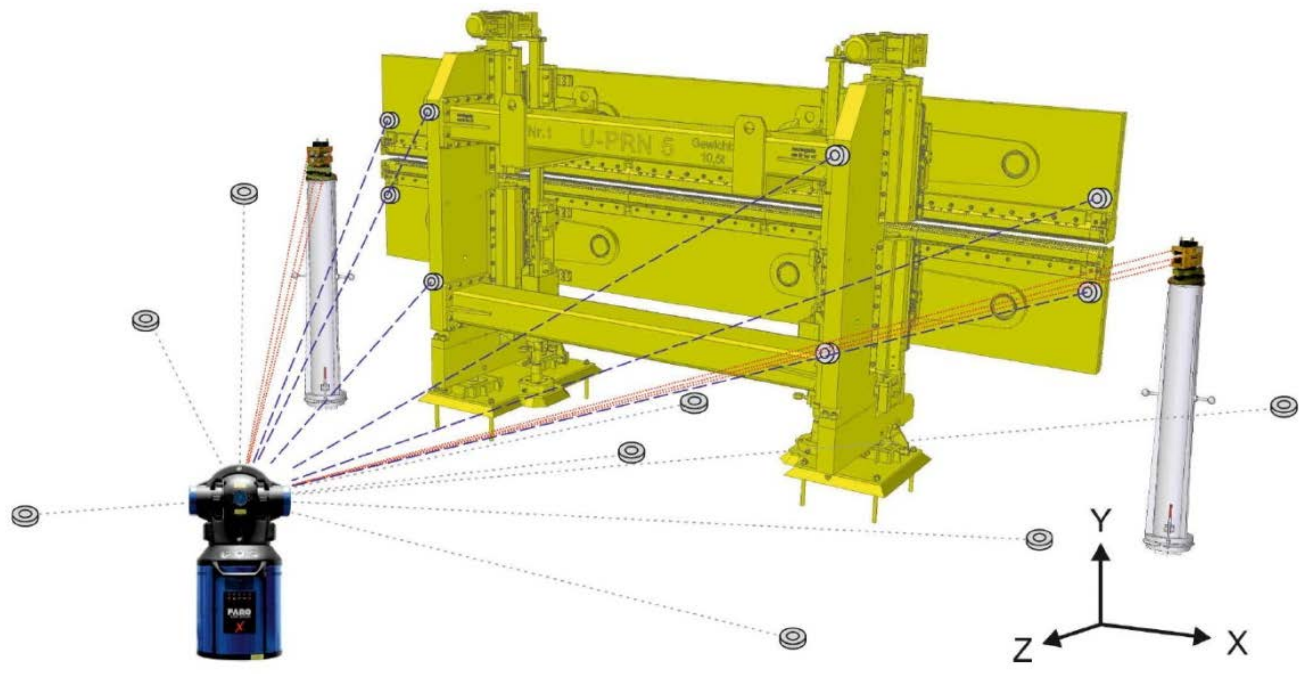

Figure 7: Placement of an undulator, two landmarks and the laser tracker for the geometrical measurements 
any kind of magnetic systems where a magnetic axis can be defined. The configuration used at EXFEL for the measurements is shown in Fig. 7. It consists of the undulator, two landmarks, one placed on either end and a Laser tracker. The coordinate convention shown in the lower right corner is the same as for the landmarks, see figures 2a), 5b). In addition there are numerous laser monuments distributed on the undulator and the floor of the surrounding lab. The Laser tracker is placed on a central point to have an optimum visibility to all monuments. It is important to mention that the measurement must be combined with the magnetic measurements and performed while the undulator is properly aligned to the magnetic measurement bench, which is not shown in figure 7 . Therefore magnetic fiducialization needs to be integrated into the magnetic measurements, which are performed on an undulator anyway. For the determination of the magnetic axis the two landmarks are placed temporarily on pillars on both sides of the undulator close to the assumed magnetic axis as shown in figure 7. They are placed within the motion range of the bench. In this position magnetic and Laser measurements can be made quasi simultaneously. In $0^{\circ}$ and $180^{\circ}$ orientation laser tracker and magnetic measurements are combined. Only transverse scans along $\mathrm{Y}$ and $\mathrm{Z}$ as shown in figure 6 need to made on the landmarks on the two positions shown in figure 7 while stability and reproducibility is provided by the tribrach support. Now, the mechanic and magnetic center positions and the transverse coordinates of the magnetic axis of the undulator can be determined as described above.

In the next step the coordinates of the monuments placed on the undulator are determined in this very coordinate system. They carry the coordinate information of the undulator and are used later for the alignment of the undulator in the tunnel using the geodetic alignment grid of monuments in the tunnels. Such an external grid of monuments was also used for the measurements in the magnetic labs. The monuments of this grid are seen in figure 7 as well. However, details of undulator alignment are beyond the scope of this paper.

\section{Accuracy considerations}

For an estimate of the alignment accuracy the influence of magnetic and mechanic measurements was investigated separately.

\section{Magnetic measurements}

Four different error sources, which have an impact on the precision of magnetic position measurements were investigated:

1. The alignment accuracy of the landmark has an impact on the zero crossings. This was discussed in section 2.2. in figures $4 \mathrm{a}$ ) and b). For a moderate $\pm 0.5 \mathrm{~mm}$ alignment $\Delta \mathrm{y}_{A l}<10 \mu \mathrm{m}$ and $\Delta \mathrm{z}_{A l}=12 \mu \mathrm{m}$ were determined, see section 2.2. In practise due to the high reproducibility of the flip tests this error might be smaller.

2. Position reproducibility, $\Delta_{P o s}$, of magnetic measurements is known from the characterization of the magnetic bench. It is the same in y and z direction. $\Delta_{P o s} \leq 5 \mu \mathrm{m}$ is assumed.

3. The accuracy of the determination of the zero crossings, $\Delta_{\text {zero }}$, as shown figure 6 depends on the quality of the data used for the linear fit to the data in normal and flipped orientation. For Y-scans the full ranges shown in figure $6 a$ ) and for Z-scans a cutout of the data over $\pm 0.5 \mathrm{~mm}$ around the zero crossings were taken. Step sizes were $0.02 \mathrm{~mm}$ for $\mathrm{Y}$ and $0.04 \mathrm{~mm}$ for Z. From the covariance analysis the slope, offset and their errors were obtained. The resulting total error on the zero crossing is given by:

$$
\Delta_{\text {Zero }}=\sqrt{2\left[\left(\frac{\Delta a}{b}\right)^{2}+\left(\frac{a \Delta b}{b^{2}}\right)^{2}\right]}
$$

Where $\mathrm{a}$ is the offset and $\mathrm{b}$ the slope and $\Delta \mathrm{a}$ and $\Delta \mathrm{b}$ the corresponding errors. The factor 2 accounts for the two independent measurements needed for the flip test. From this analysis for Y-scans the observed total error amounts to $\Delta y_{Z \text { Zero }}=1 \mu \mathrm{m}$ and for Z-scans to $\Delta z_{Z e r o}=24 \mu \mathrm{m}$. The difference of the errors originates from the better linearity of the Y-data as compared to Z-data.

4. Any external magnetic field such as an ambient magnetic field superimposes to the field of the PM quadrupole and might shift the zero crossing. This shift, $\Delta_{B}$, by a field change $\Delta B$ is given by:

$$
\Delta_{B}=\frac{\Delta B}{C}
$$


$\mathrm{C}$ is the field gradient. For a worst case estimate $\mathrm{C}$ was evaluated for the skew quadrupole to be $0.075 \mathrm{~T} / \mathrm{mm}$ and a full swap of the ambient field in Hamburg from $-40 \mu \mathrm{T}$ to $+40 \mu \mathrm{T}$ was assumed. This would lead to a position change of $\pm 0.5 \mu \mathrm{m}$ only, so that this effect is negligible.

The resulting total errors from magnetic measurements is the square sum of the above and results in:

$$
\Delta_{\mathrm{Mag}, Y}=11 \mu \mathrm{m} \text { and } \Delta_{\mathrm{Mag}, Z}=27 \mu \mathrm{m}
$$

This error estimate is conservative and assumes statistically independent errors. The largest contributions come from points 1 and 3 above and both include flip tests where some errors might partially compensate. So total errors might be smaller. Meanwhile the landmark is used routinely and there is plenty of data, which are used to evaluate the overall repeatability of the measurements. This gives information about a combined effect of the above. While the mean between two magnetic measurement values from orientation 1 and orientation 2 gives the true magnetic axis for this landmark, see eq. (5), the difference between orientation 1 and orientation 2 is a characteristic number, which should not change with time:

$$
Y, Z_{\text {Cent }, \text { Mag }}=\left(Y, Z_{\text {Mag, } N}-Y, Z_{\text {Mag, },}\right)
$$

The results for a measurement series over more than two years is shown in figure 8. The standard deviation is in the range of $5-13 \mu \mathrm{m}$, which is in good agreement with the above estimates.

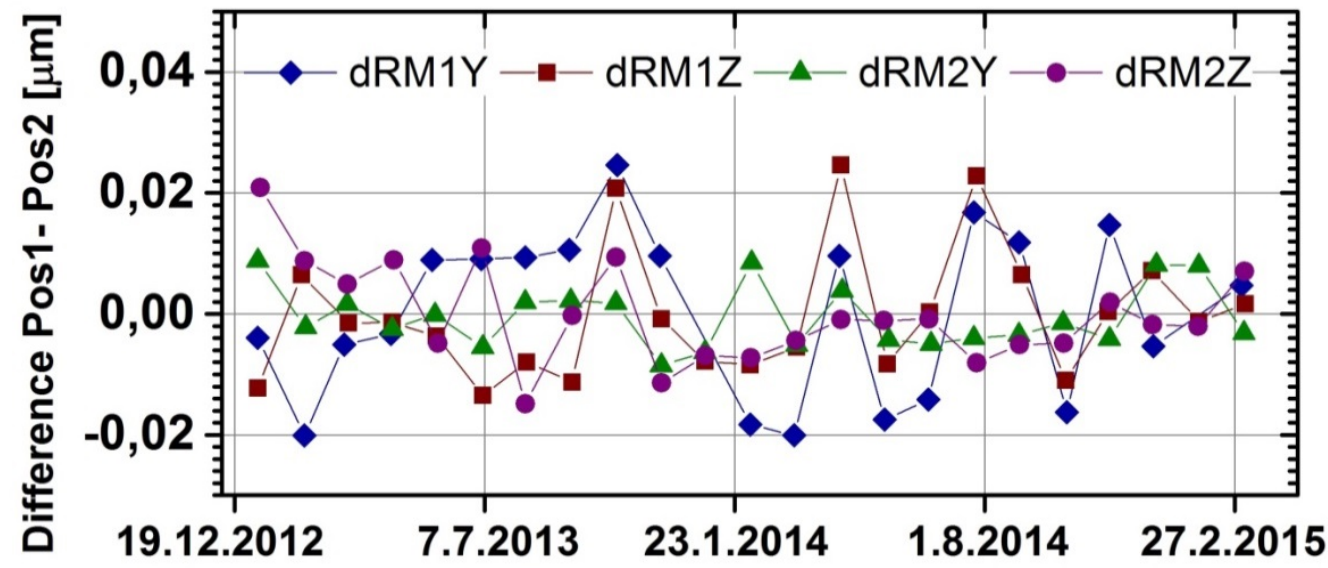

Figure 8: Repeated differences between orientation 1 and orientation 2 of landmark1 (RM1) and 2 (RM2)

\section{Laser Tracker measurements}

Point determination of a laser tracker is done by measuring 2 angles and one distance (3D polar coordinates) at the same time. There are various error sources that have to be considered when looking at laser tracker measurements, e.g. angular measurement, distance measurement, synchronization of measurements, temperature, air pressure, humidity, centering error of target, etc.. The measurement on undulators were done in an air conditioned environment and distances were below 5 meters, which increases accuracy. Under these conditions the general accuracy of a single point measurement is given by:

$$
\sigma_{P}=\sqrt{\sigma_{x}^{2}+\sigma_{y}^{2}+\sigma_{z}^{2}}<0.05 \mathrm{~mm}
$$

It should be noted, that this is a conservative approach, given the overall accuracy of an arbitrary point in a 10 meter-3D space. The relative error between two adjacent points measured consecutively is generally much smaller. Since the position of the landmark itself is determined by measuring at least 10 points, the position of the mark is known to better than

$$
\sigma_{M}=\frac{\sigma_{P}}{\sqrt{n-1}}<17 \mu m
$$

where $\mathrm{n}$ is the number of measurements. All other mechanical imperfections of the mark itself are eliminated by the two-face measurement and have not to be considered here. 
Determination of the Undulator position is done in a similar way. Up to 11 individual fiducials are distributed well over the volume of the Undulator and thus form a grid of fiducials which are used for the in situ alignment in the tunnels later on. These fiducials are also measured with the same laser tracker during the fiducialization of the Undulator. With the same conservative approach as for the accuracy estimation above

$$
\sigma_{U g}<16 \mu m
$$

is obtained for the accuracy of the geometric Undulator position during the fiducialization process.

\section{Total accuracy}

Combining the accuracy of the magnetic measurements together with the geometric measurement, using the law of error propagation the overall accuracy for the Undulator position related to the magnetic axis is obtained

$$
\sigma_{U m}=\sqrt{\Delta_{A l}^{2}+\Delta_{P o s}^{2}+\Delta_{Z e r o}^{2}+\sigma_{M}^{2}+\sigma_{U g}^{2}}
$$

resulting in an accuracy of $\pm 26 \mu \mathrm{m}$ for $\mathrm{Y}$ and $\pm 36 \mu \mathrm{m}$ for $\mathrm{Z}$.

\section{Summary}

In this paper, a magnetic landmark is described, which allows the accurate transformation of magnetic coordinates to geometrical coordinates obtained from a Laser Tracker system.

The method was developed for the precision alignment of undulator segments in the EXFEL tunnels with respect to their magnetic axes. It is used for the transfer measurements which are done while an undulator is still in the magnetic lab and fully aligned to the magnetic bench. With the help of the landmark the magnetic axis is transferred to a set of laser fiducials on the undulator segment. So the alignment in the tunnels can be done by the geometric alignment using Laser Trackers. The combined overall accuracy is estimated to be $\pm 26 \mu \mathrm{m}$ in $\mathrm{Y}$ and $\pm 36 \mu \mathrm{m}$ in $\mathrm{Z}$. The method is used for transfer measurements of the 91 undulator segments for the EXFEL.

\section{References}

[1] M. Altarelli et al., “The European X-ray Free-electron Laser”, Technical Design Report, ISBN 3-935702-17-5, 2006; available under: http://www.xfel.eu/documents/technical documents

[2] LCLS II Design report, SLAC-R-978, 2011; available under: http://www.slac.stanford.edu/cgi-wrap/getdoc/slac-r-978.pdf

[3] Z. Wolf, V. Kaplunenko, Y. Levashov, A. Weidemann, ”LCLS Undulator Tuning and Fiducialization”, Proceedings of the PAC 07, June 25-29, 2007, Albuquerque, New Mexico, USA

[4] Y. Levashov, Z. Wolf, "Test of coordinate transfer from magnetic to mechanical reference for LCLS undulator fiducialization”, SLAC Report LCLS-TN-05-10; available under: http://www-ssrl.slac.stanford.edu/lcls/lcls_tech_notes.html

[5] J. Pflueger and G. Nawrath, European XFEL WP71 Internal Report No: WP71/2011/22 (2011), unpublished.

[6] R. Tatchyn, Nuclear Instruments and Methods A341 (1994), 449-453. 